Klaus Schabacker

\title{
Die moderne ökonomische Theorie und die Kapitaltheorie von Marx*
}

\section{Einleitung}

Auf den ersten Blick tragen die unterschiedlichen und weit auseinanderliegenden Urteile über die Marxsche ökonomische Theorie in einem Grade zur Verwirrung bei, daß die Frage aufkommt, ob tatsächlich derselbe Autor gemeint ist. Leontief beispielsweise schätzt an Marx dessen gründliche empirische Kenntnisse: »His strength lies in realistic, empirical knowledge of the capitalist system « (Leontief 1938, 8). Ganz anders dagegen Morishima; er würdigt Marx - vielleicht mit einem Augenzwinkern - als einen mathematischen Ökonomen, auch wenn dieser sich höchst selten um formale Darstellungen bemüht habe (Morishima 1974, 612). Samuelsons Urteil fällt hingegen weniger freundlich aus: In einer frühen Schrift hielt er Marx für einen minor Ricardian, dessen Preistheorie nicht überzeugen könne. Joan Robinson, Oskar Lange und Paul Sweezy gewichten den Marxschen Beitrag zur ökonomischen Theoriebildung wieder anders: Sie vertreten die Auffassung, daß man die Preistheorie getrost der Neoklassik überlassen könne, die für diese Zwecke besser gerüstet sei. So kritisiert Lange die Marxsche Werttheorie als ein antiquiertes Gleichgewichtsmodell, dem das walrasianische System vorzuziehen sei, und Joan Robinson argumentiert, daß die Arbeitswerttheorie für eine Analyse der kapitalistischen Ökonomie und mithin für das, was Marx eigentlich sagen wollte, überflüssig sei. ${ }^{1}$ Marxens hauptsächliche Leistungen erkennen sie in seiner Untersuchung der Einkommensverteilung auf die Klassen, in seiner Analyse der Akkumulation und der Entwicklung der kapitalistischen Produktion. Der Vorzug und die Überlegenheit der Marxschen Theorie gründe darin, $\mathrm{da} ß$ er die sozialen und institutionellen Bedingungen der ökonomischen Dynamik in seine Betrachtung einbeziehe, und Joan Robinson betont, daß Marx die historische Zeit als eine wesentliche Eigenschaft dieses ökonomi-

* Für kritische Diskussionen und wichtige Hinweise danke ich Michael Heinrich.

1 Lange (1934/35, 195f); Robinson (1942, 27); Sweezy (1942, 156); Samuelson (1957). Siehe auch Dobb (1973, 159-161) für weitere Urteile über die Marxsche Theorie. 
schen Systems erkannt und in seinen Analysen berücksichtigt habe. Ganz ähnlich sieht Schumpeter den Marxschen Beitrag zur ökonomischen Theoriebildung in dessen intensiver Beschäftigung mit den langfristigen Entwicklungslinien der kapitalistischen Produktion und den beständigen Veränderungen, die sie mit sich bringt; in diesem Zusammenhang verweist auch Schumpeter auf die historische Zeit, die in Marxens Analyse Eingang gefunden habe. ${ }^{2}$ Diese zuletzt genannten Interpretationen kommen der Marxschen Intention sicherlich sehr nahe, denn Marx betont von Anbeginn und mit großem Nachdruck, daß er die ökonomischen Beziehungen innerhalb bestimmter historischer Konstellationen untersuche, so daß immer auch die Institutionen und die Machtverteilung in einer Gesellschaft thematisiert werden. Die Warenproduktion wird, wie Sweezy $(1942,39)$ es formuliert, »zum Gegenstand soziologisch-historischer Untersuchung«. Marx behandelt diese Probleme in einem theoretischen Rahmen, in dem häufig makroökonomische und mikroökonomische Analysen ineinander fließen. Daneben gibt es in seiner Analyse aber auch eine andere Schicht, in der vor allem die reine Preis- und Verteilungstheorie zu finden sind: Marx benutzt disaggregierte Modelle, um die Gleichgewichtslösungen seiner ökonomischen Systeme zu untersuchen. In seiner Kapitaltheorie steckt immer auch eine Gleichgewichtstheorie, obgleich seine Untersuchung nie vollständig darin aufgeht. So betont Sweezy in seiner umfangreichen Auseinandersetzung mit dem Marxschen Kapital, daß das Wertgesetz eine allgemeine Gleichgewichtstheorie enthalte. ${ }^{3}$ Gut dreißig Jahre später rückt Morishima in seinem Buch: Marx's Economics dieses Modell des allgemeinen Gleichgewichts in den Mittelpunkt seiner Interpretation und vertritt die - an der hergebrachten theoriegeschichtlichen Einteilung gemessen - unorthodoxe Auffassung, wonach Ricardo, Marx und Walras die Vorläufer und Begründer der allgemeinen Gleichgewichtstheorie gewesen seien; sie erarbeiteten im 19. Jahrhundert die Grundlage für die moderne ökonomische Theorie, und Marxens Leistungen in dieser Hinsicht stünden denen von Walras in nichts nach. ${ }^{4}$ Mit dieser Sichtweise der theoriegeschichtlichen Zusammenhänge setzt Morishima sich ebenso souverän über die gewöhnliche Unterscheidung zwischen einer objektivistischen und einer subjektivistischen Werttheorie hinweg wie über die Klassifizierung nach Surplustheorien und Grenzproduktivitätstheorien der Verteilung. Pasinetti hingegen, der ebenso wie Morishima disaggregierte Gleichgewichtsmodelle verwendet, gelangt zu einer ganz anderen Einteilung. Er unterscheidet das Produktionsparadigma vom Tauschparadigma (Pasinetti 1993, XIVf); das erste beruhe auf

2 Siehe Lange (1934/35, 194); Schumpeter (1942, 78); Sweezy (1942, 39); Robinson (1979, 90).

3 Sweezy $(1942,71)$ und ähnlich Meek (1956, XVII) in einem Vorwort zur zweiten Auflage seines Buches im Jahr 1973.

4 Morishima (1973, 1-3); Morishima (1974) sowie Morishima (1989, 1, 13). 
dem Prinzip des Lernens, i.e. auf dem technischen Fortschritt und der Entwicklung der Produktivität, das zweite hingegen auf den Prinzipien des Nutzens und der Optimierung. Demzufolge wird die Walrassche Ökonomik dem Tauschparadigma zugeordnet, wohingegen Ricardo und Marx als Ökonomen begriffen werden, die im Rahmen des Produktionsparadigmas Vorarbeiten für eine Theorie des strukturellen Wandels geleistet hätten (ebd., 11). Die Antwort auf die Frage, welchen Platz Marx in der Geschichtsschreibung der Ökonomik einnimmt, hängt also von der Beantwortung der vorgelagerten Frage ab, welche Problemstellungen für die ökonomische Theoriebildung als konstitutiv angesehen werden.

Auf der anderen Seite zeigen solche divergierenden Einschätzungen aber auch, daß Marxens Kapitaltheorie in vielen Hinsichten mit der Entwicklung des ökonomischen Denkens verwoben ist. Im folgenden werden wir von der Dynamik, der Konjunkturtheorie und der historischen Zeit absehen und in einem - verglichen mit dem thematisch weitgespannten Bogen der Marxschen Untersuchungen ${ }^{5}$ - deutlich eingeschränkten Rahmen der Frage nachgehen, worin Marx Beitrag zur ökonomischen Theoriebildung gesehen wird. Wir legen dabei Gleichgewichtsmodelle zugrunde. Eine solche Untersuchung der Marxschen Kapitaltheorie im Rahmen von Gleichgewichtsmodellen erfaßt diejenigen Schichten in seiner Argumentation nicht, in denen Marx eine Kritik an den Kategorien entwickelt, in denen die ökonomischen Theoretiker wirtschaftliche Zusammenhänge begreifen und darzustellen suchen. Allerdings wurden immer wieder gerade die Gleichgewichtsmodelle benutzt um zu demonstrieren, daß die Marxsche Preistheorie vor unlösbaren Problemen steht. Greift man die Theorie des allgemeinen Gleichgewichts auf, die im Marxschen Kapital enthalten ist, so kann man zeigen, daß diejenigen Schwierigkeiten, welche als Besonderheiten der Marxschen Arbeitswerttheorie hervorgehoben wurden, tatsächlich allgemeiner Natur sind und in jeder Kapitaltheorie auftreten.

Im folgenden werden wir zunächst den Einfluß Ricardos auf das ökonomische Denken seiner Nachfolger betrachten (2), um dann auf Marx Beitrag zur ökonomischen Theoriebildung einzugehen. Dabei untersuchen wir seinen Gleichgewichtsbegriff (3) und sein Modell der industriellen Verflechtungen (4). Im 5. Abschnitt werden in einer theoriegeschichtlichen Perspektive die Beziehungen skizziert, die zwischen der Struktur der gesellschaftlichen Produktion, der Preisbestimmung und der Einkommensverteilung hergestellt wurden; auf den Zusammenhang zwischen Geld und Kapitalvorschüssen wird im 6. Abschnitt eingegangen. Den Abschluß (7) bildet eine kurze Betrachtung über die verteilungsinduzierten Umbewertungen.

5 In einem weiter gefaßten Spektrum von Fragestellungen behandelt in jüngerer Zeit Levine (1995) die Marxschen Themen. 


\section{Das Ricardianische Erbe}

Ricardos Principles of Economics haben die ökonomische Theoriebildung nachhaltig beeinflußt. Ihr Thema ist die Preisbestimmung, die Einkommensverteilung und die Akkumulation. Ricardos Fragestellungen, seine Einteilung des Gegenstandes und sein auf die Bildung ökonomischer Modelle gerichtetes methodisches Vorgehen wirkten beispielgebend für das moderne ökonomische Denken. ${ }^{6}$ Ricardo beginnt seine Preistheorie mit dem Modell eines einfachen Gemeinwesens, in dem es kein Kapital gibt und der Boden im Überfluß vorhanden ist. Unter diesen Bedingungen werden die Austauschverhältnisse der Waren durch die in ihnen aufgespeicherten Arbeitsmengen reguliert; die Verteilungsregel ist denkbar einfach, denn das Einkommen eines jeden Produzenten hängt von der individuell geleisteten Arbeitsmenge ab. Sobald jedoch das Kapitaleigentum und die Lohnarbeit in die Analyse einbezogen werden, stellt sich für dieses neue Modell die ungleich schwierigere Frage, in welcher Weise die Verteilung des Einkommens auf den Lohn und den Profit die relativen Preise der Waren beeinflußt. Ricardo ist der erste, der dieser Frage nachgeht (Ricardo 1817, 22, Fn. 3), und er findet heraus, daß die Preisverhältnisse der Waren wechseln, wenn der Reallohnsatz steigt oder fällt. Diese Verschiebungen im Preissystem werden immer dann eintreten, wenn die Industriezweige eine unterschiedliche Zusammensetzung aus Kapital und Arbeit aufweisen. Wie seine Untersuchung zeigt, ist der Gleichgewichtsbegriff für das Modell einer kapitalistischen Ökonomie komplizierter als der des einfachen Gemeinwesens, denn die Warenpreise müssen in einem solchen Verhältnis zueinander stehen, daß jeder Produktionszweig sowohl seine physischen Einsatzmengen ersetzen als auch die allgemeine Profitrate realisieren kann. Ricardos ökonomische Theorie ist durchaus verwickelt. Die Einkommensverteilung und somit der Reallohnsatz und die Profitrate treten zweimal auf: das erste Mal in der Theorie der Preisbestimmung, die zeigt, daß die relativen Preise von einer Verteilungsgröße abhängen, und das zweite Mal im Zusammenhang mit der Akkumulation des Kapitals, dem Wachstum der Bevölkerung und der Ausdehnung der landwirtschaftlichen Produktion auf schlechtere Böden.

In den groben Zügen folgt der Aufbau der Marxschen Preistheorie dem Vorbild Ricardos. Allerdings fügt Marx noch eine Zwischenstufe ein, die bei Ricardo fehlt. Bevor er im dritten Band auf die Produktionspreise zu sprechen kommt, benutzt er einen Kunstgriff, um die Schwierigkeiten aus dem Weg zu räumen, die aus der Verbindung der Preistheorie mit der Ver-

6 Schon bei Adam Smith (1776) finden sich die Ansatzpunkte einer solchen Gliederung, allerdings fehlt ihm die methodische Strenge in der Analyse und seine Theorie der relativen Preise bricht mit der Einführung einer allgemeinen Profitrate ab. 
teilungstheorie erwachsen. Er führt die Mehrwertrate als Verteilungsregel für die Kapitaleigentümer ein, so daß der Überschuß respektive der Profit proportional zum Vorschuß für die Löhne verteilt wird. Dergestalt vom Transformationsproblem abgeschirmt, konzipiert Marx das Mehrwertmodell, worin die Austauschverhältnisse der Waren weiterhin durch die Arbeitsmengen reguliert werden, das Einkommen aber in den Reallohn und den Mehrwert zerfällt. Dieses Modell vereint die Arbeitsmengentheorie der Preise als Eigenschaft des einfachen Modells mit der Existenz von Klassen, die charakteristisch für das Profitmodell ist. Die Marxsche Preistheorie kann mithin in drei Ebenen eingeteilt werden, auf denen jeweils bestimmte Fragestellungen bearbeitet werden. Marx benutzt das einfache Modell, um den Austausch der Waren, das Geld, den Geldgebrauch sowie die Geldfunktionen zu betrachten. Das Mehrwertmodell trägt eine erhebliche Begründungslast, denn er entwickelt auf dieser Grundlage den Produktionsprozeß in der modernen Fabrik, die Wirkung der steigenden Produktivität neuer Produktionsverfahren auf die Preise und die Einkommensverteilung, die Akkumulation des Kapitals und schließlich die Kreislaufbeziehungen in der Ökonomie. Das Profitmodell ist am wenigsten ausgearbeitet; es dient der Analyse der Konkurrenz, des Zinses und des Kredits und liegt Marxens Überlegungen zum tendenziellen Fall der Profitrate zugrunde.

Neben Marx greifen auch die frühen neoklassischen Ökonomen wie Walras, Jevons, Böhm-Bawerk und Wicksell ein von Ricardo geprägtes Schema der Untersuchung auf. Sie beginnen ihre Analysen ebenfalls mit einem einfachen Modell, um die Grundzüge der Preistheorie zu entwickeln, und gehen in einem anschließenden Teil auf das kompliziertere Modell einer kapitalistischen Ökonomie ein, worin das Vorhandensein von Kapital zu Verwicklungen der Preistheorie mit der Verteilungstheorie führt. Allen genannten Autoren ist der Gedanke gemeinsam, daß mit dem Kapital und dem Profiteinkommen ein neues Element in die ökonomische Theorie aufgenommen wird, das sich nicht in einfacher Weise in das ursprüngliche Modell einfügen läßt. Allerdings sollte dieses gemeinsame Schema nicht überspannt werden: Unter den Autoren herrschen die größten Differenzen darüber, was unter dem einfachen Modell des ursprünglichen Gemeinwesens und unter dem Modell einer kapitalistischen Ökonomie zu verstehen sei. Marxens »einfache Warenzirkulation« dient der Bestimmung der Preise aus den in der Produktion aufgewendeten Arbeitsmengen, während die frühen neoklassischen Ökonomen in ihren einfachen Modellen von der Produktion vollständig absehen und die Preise ausschließlich aus dem Austausch gegebener Güterbestände entwickeln. Ricardo und Marx begreifen das Kapital als eine Wertgröße und charakterisieren die kapitalistische Ökonomie durch die sozialen Verhältnisse, welche die Produzenten als Kapitaleigentümer und Arbeiter eingehen. Anders argumentieren die neoklassi- 
schen Ökonomen, obgleich sie in dieser Frage keine einheitliche Auffassung vertreten. Böhm-Bawerk, der - vielleicht entgegen den eigenen Absichten - eine Grenzproduktivitätstheorie der Verteilung ausarbeitet, betrachtet das Kapital als eine Menge von Produktionsmitteln, genauer: als eine Menge von Zwischenprodukten, die zur Herstellung von Konsumgütern eingesetzt wird (Böhm-Bawerk 1888, 141). Wie er sehr anschaulich beschreibt, ist die »kapitallose Produktion « demgegenüber eine solche, »die geradeaus mit der nackten Faust auf das Ziel zugeht« (ebd., 16). Die kapitalistische Produktion erfordert also zeitaufwendige Produktionsumwege. ${ }^{7}$ Diese Position wird von der österreichischen Schule, von Wicksell und Jevons vertreten und begründet einen Strang des neoklassischen Denkens. Eine andere Linie verfolgt Walras. Er unterscheidet vier Gleichgewichtsmodelle, nämlich das Tauschgleichgewicht, das Produktionsgleichgewicht, die Kapitalbildung und schließlich das Modell mit Vorprodukten und Geldhaltung. Zu den Kapitalgütern im engeren Sinne (capital proper) zählt er nur solche produzierten Produktionsmittel, welche für längere Zeit im Produktionsverfahren eingesetzt werden und langsam verschleißen (Walras 1874, 212 und 217). Es fällt zunächst schwer, in der Walrasschen Theorie zwischen einer kapitalistischen und einer nichtkapitalistischen Produktion zu unterscheiden. Anders als bei der österreichischen Schule bietet die Existenz produzierter Produktivgüter für ihn kein Unterscheidungskriterium, weil diese Produktionsmittel als Bestandteil der Erstausstattung bereits in das Produktionsgleichgewicht eingehen; eine kapitallose Produktion ist hier nicht vorgesehen. ${ }^{8}$ Wenn Eatwell $(1987,868)$ dennoch zwischen einer nichtkapitalistischen Produktion, die im Produktionsgleichgewicht dargestellt wird, und dem Modell der kapitalistischen Produktion bei Walras unterscheidet, das im Rahmen der Kapitalbildung eingeführt wird, dann spielt auch hier die Einkommensverteilung wieder die entscheidende Rolle. Walras $(1874,267-77)$ untersucht den Zins erst im Zusammenhang

7 Böhm-Bawerk $(1888,111 \mathrm{f})$. Auf der anderen Marktseite wird das Zeitelement durch die Zeitpräferenz der Haushalte eingeführt.

8 An dieser Stelle unterscheidet sich Walras deutlich von der österreichischen Schule. Die letztere entwickelt das Tauschgleichgewicht unter der Voraussetzung, daß gegebene Bestände von Konsumgütern vorhanden sind, sobald aber die kapitalistische Produktion untersucht wird, durchbricht sie dieses methodische Prinzip und ergänzt die Erstausstattung durch das Zeitelement: die Produktivgüter werden in einem zeitaufwendigen Produktionsverfahren hergestellt, an dessen Anfang nur die Ressourcen Arbeit und Boden stehen. Eine Anfangsausstattung gibt es hier nur für diese Ressourcen - sowie für die Konsumgüter -, jedoch nicht für die Kapitalgüter. Auf der anderen Seite hat das Walrassche System keinen Platz für das Zeitelement. In seiner Produktionstheorie wird dies sinnfällig: Walras führt sogenannte tickets ein, welche die angebotenen und nachgefragten Gütermengen repräsentieren und dazu dienen, die Produktion und den Tausch zu Ungleichgewichtspreisen auszuschließen (Walras 1874, 242). Auf diese Eigenheit des Walrasschen Allokationsmodels verweist Pasinetti $(1975,48)$ und kritisiert, daß der Produktionsstrom einer Periode auf diese Weise in einen Vorrat - also in eine Bestandsgröße - verwandelt werde. 
mit der Produktion zusätzlicher Kapitalgüter und dem Gleichgewicht zwischen Sparen und Investieren. Auf diese Weise kommt ein ungewöhnliches Bild zustande: eine kapitalistische Produktion gibt es nur, wenn die Ökonomie akkumuliert, während die entscheidenden Personengruppen: die Arbeiter, die Kapitaleigentümer und die Unternehmer schon im Modell der nichtkapitalistischen Produktion (ebd., 222) vorhanden sind.

Darüber hinaus teilen Ricardo, Marx, Walras, Jevons, Böhm-Bawerk und Wicksell die Auffassung, daß es eine einheitliche Profitrate respektive Zinsrate auf das vorhandene Kapital gibt. Sie stehen vor dem gemeinsamen Problem zu erklären, wie eine solche einheitliche Rate zustandekommt. Vermeintlich sind die klassischen Ökonomen zunächst im Vorteil, weil sie das Kapital als eine Wertgröße begreifen. Ist die Menge der benutzten Produktionsmittel gegeben, so scheint - ihren Überlegungen zufolge - der Kapitalwert der Produktionsmittel eine Konstante zu sein, die, neben der Lohnsumme, einem größeren oder geringeren Profit zur Bemessungsgrundlage dient, so daß aus diesem Verhältnis zweier Wertgrößen die allgemeine Profitrate entsteht. Ein solches Verfahren zur Bestimmung der Profitrate ist jedoch ebenso trügerisch wie dasjenige, das die neoklassischen Autoren anwenden. ${ }^{9}$ Von denjenigen Ökonomen, die eine Grenzproduktivitätstheorie der Verteilung vertreten, hat Wicksell die Schwierigkeiten am klarsten erkannt und herausgearbeitet, die dieser Verteilungstheorie entgegenstehen. Anders als die klassischen Autoren sucht er einen Weg, um den Lohn und den Profit als Einkommen der Produktionsfaktoren aus deren technisch bestimmten Beiträgen zum Produktionsergebnis - aus den Grenzproduktivitäten - abzuleiten. Einheitliche Entlohnungssätze existieren daher nur für homogene physische Einsatzmengen. Wie in der klassischen Theorie nehmen auch die neoklassischen Autoren an, daß die Arbeit homogen ist, und erhalten sodann einen einheitlichen Reallohnsatz. Beim Kapital wird die Sache verwickelter ${ }^{10}$ : Geht man von einem physischen Kapitalbegriff aus, dann erhält man ebensoviele Profitraten, wie es verschiedenartige Kapitalgüter - Produktionsmittel also - gibt. Somit wird, wie Wicksell $(1913,213)$ zutreffend feststellt, »eine einheitliche Betrachtung der Rolle des Kapitals in der Produktion (...) unmöglich «. In dieser Formulierung steckt in der Tat das eigentliche Problem: Wicksell sucht nach einer Symmetrie zwischen der Verteilung und der Produktion, derzufolge das Kapital in beiden die gleiche, nämlich einheitliche Rolle spielt. Bei der Suche nach einer solchen 'einheitlichen Rolle des Kapitals in der Produktion' folgt man jedoch

9 Es ist nur für den besondere Fall richtig, in dem alle Produktionszweige die gleiche Zusammensetzung aus Kapital und Arbeit aufweisen.

10 Die Schwierigkeiten entstehen aber nicht daraus, daß mehr als ein Kapitalgut eingesetzt wird, sondern daraus, daß die Ökonomie überhaupt mehr als ein Gut produziert (Bliss 1975, 85). - Die Annahme, daß die Arbeit homogen sei, ist willkürlich gewählt. 
einem Irrlicht, weil es sie in den technischen Produktionsverfahren nicht gibt. Wicksell benötigt den Wert aller Produktionsmittel, »um den allem Kapital bei wirtschaftlichem Gleichgewicht gemeinsamen Zinsfuß zu berechnen « (ebd.). Daran wird das Dilemma deutlich, in dem sich die neoklassische Grenzproduktivitätstheorie der Verteilung befindet: Will man die Einkommen der Produktionsfaktoren aus ihren Grenzproduktivitäten ableiten, dann müssen sie als physische Mengeneinheiten in die Betrachtung aufgenommen werden; auf diese Weise erhält man eine Vielzahl individueller Grenzproduktivitäten, aber keine einheitliche Profitrate. Um die letztere bestimmen zu können, muß an die Stelle der heterogenen Kapitalgüter eine homogene Wertgröße: der Kapitalwert gesetzt werden. Eine solche Größe hat jedoch im Produktionsverfahren keine Bedeutung. Um eine einheitliche Rate der Grenzproduktivität zu ermitteln, die als Verteilungsregel für das Kapital brauchbar wäre, stellt sich für Wicksell das Problem, aus den heterogenen Kapitalgütern eine homogene physische Menge zu konstruieren, die als Kapital interpretiert werden kann. Die vermeintliche Lösung des Problems findet er darin, daß alle Produktionsmittel die Eigenschaft haben, Arbeitsprodukte zu sein, und daher in aufgespeicherte Arbeitsmengen verwandelt werden können. ${ }^{11}$ Zudem werden diese Arbeitsmengen datiert, um den Zeitraum zu erfassen, der verstreichen muß, bis sie schließlich zusammen mit den Vorprodukten, in denen sie enthalten sind, in das Endprodukt eingehen. Man erhält somit das auf den ersten Blick überraschende Ergebnis, daß klassische Ökonomen wie Marx und Ricardo den Wert des Kapitals ganz ebenso aus den Arbeitsmengen ableiten wie der neoklassische Theoretiker Wicksell. Auch bei diesem Problem schlägt Walras einen anderen Weg ein. Wie bereits erwähnt, fällt seine Kapitaltheorie mit der Wachstumstheorie zusammen. Im Gegensatz zu Marx ist die Zinsrate respektive die allgemeine Profitrate bei Walras eine abhängige Variable, die zusammen mit den übrigen Unbekannten: den Preisen und den Mengen bestimmt werden muß. Die Schwierigkeiten treten indes an anderer Stelle auf. Walras $(1874,273 f)$ führt ein imaginäres, homogenes Spargut ein, das die Haushalte ebenso nachfragen wie die anderen Güter. In sein disaggregiertes Modell wird eine aggregierte Größe - das Sparen - eingelassen, die eher für makroökonomische Argumentationen kennzeichnend ist, wie Morishima $(1977,6)$ bemerkt. Nun erhält das Kapital zwar weder in der Produktion noch bei den Investitionen in zusätzliche Produktivgüter eine einheitliche Rolle, denn diese Teile der Walrasschen Analyse formen das disaggregierte Modell, aber bei der Einkommensverteilung schlägt das einheitliche Spargut die Brücke zur einheitlichen Zinsrate. Wie Eatwell $(1987,872)$ zeigt, ist eine konsistente Lösung für das Gleichungssystem

11 Wicksell $(1913,214)$. Von den aufgespeicherten Bodenleistungen sehen wir hier ab. 
nur unter der sehr einschränkenden Bedingung sichergestellt, daß genau ein Kapitalgut erzeugt wird (vgl. auch Kurz und Salvadori 1995, 439-41).

Alle Autoren, mit der Ausnahme von Walras, stehen vor der gleichen Schwierigkeit, wenn die allgemeine Profitrate ermittelt werden soll: Sie setzen eine von der Einkommensverteilung unabhängige Konstante voraus, die für eine bestimmte Menge der verwendeten Produktionsmittel steht, und deren Zahlenwert nur dann wechseln soll, wenn die benutzten Produktivgütermengen variieren. Stets handelt es sich darum, eine heterogene Gütermenge zu homogenisieren. Sie vernachlässigen dabei Ricardos Erkenntnis, daß die Preise von der Einkommensverteilung abhängen. In den modernen ökonomischen Theorien hat dieses Problem zwei verschiedenartige Lösungen gefunden. Die eine wird von Sraffa (1960) und den Neoricardianern vertreten; demnach werden das Preissystem und eine VerteilungsgröBe - der Reallohnsatz oder die Profitrate - simultan determiniert, wenn die Produktionstechnik und die jeweils andere Verteilungsgröße bekannt sind. Die andere Lösung findet man im Rahmen der allgemeinen Gleichgewichtstheorie. Sie kann auf die Voraussetzung verzichten, daß ein Verteilungsparameter gegeben ist, und leitet sowohl die relativen Preise als auch den Lohnsatz in einem System der intertemporalen Allokation bei gegebener Erstausstattung ab. Bei dieser Lösung gibt es jedoch im allgemeinen keine einheitliche Profitrate mehr (Bliss 1975, 53).

\section{Die Gleichgewichtstheorie}

Marx sucht in allen drei Modellen - in der einfachen Warenzirkulation, im Mehrwert- und im Profitmodell - eine allgemeine Gleichgewichtslösung für die Einkommensverteilung und das Preissystem. Bereits in der 'einfachen Warenzirkulation' führt er die Grundzüge seines Gleichgewichtsbegriffs aus. Erstens: Das sogenannte Wertgesetz besagt, daß die Warenpreise durch die aufgespeicherten Arbeitsmengen, i.e. die Arbeitswerte, bestimmt werden (Marx 1867, 54). Marx untersucht die Gesetze, nach denen der Warenaustausch unter normalen Bedingungen vollzogen wird. In diesem $\mathrm{Zu}$ sammenhang bedeutet normal, daß von den Sonderkonjunkturen des Marktes und den zufälligen Schwankungen der Marktpreise abgesehen wird (siehe Marx 1867, 122). Beginnend mit dem Austausch zweier Waren weitet er die Untersuchung aus und bezieht schließlich alle Waren ein. Marx verfolgt von Anfang an eine Totalanalyse der Märkte - in seiner Terminologie: die Beziehungen innerhalb der »Warenwelt« (Marx 1867, 80). Zweitens: Marx bleibt nicht bei den unüberschaubar vielen Preisrelationen zwischen jeweils zwei Waren stehen, sondern vereinfacht das System der Tauschwerte, indem er einen einheitlichen Wertstandard einführt - das »allgemeine Äquivalent«. Drittens: Da es in diesem Gemeinwesen keine 
Klassen gibt, wirft die Einkommensverteilung keine Probleme auf; wie bereits angeführt, ist die Verteilungsregel sehr einfach, denn die Höhe des Einkommens richtet sich nach der individuellen Arbeitsleistung. Viertens: Marx unterstellt eine arbeitsteilige Gesellschaft, die sich nur dadurch erhalten kann, daß die »Privatproduzenten« ihre Waren austauschen. Auf dieser Grundlage sind der Austausch und die Preisbildung keine zufälligen oder ephemeren Erscheinungen, sondern sie entstehen zwangsläufig und sind dauerhaft; der Austausch ist eine regelmäßige ökonomische Beziehung zwischen den Produzenten (Marx 1867, 120). Ferner ist dieser Austausch umfassend: Es werden nicht nur Konsumgüter, sondern auch Produktivgüter gehandelt (Marx 1867, 120). Marxens Rede von der »Verschlingung der Warenkreisläufe « (Marx 1867, 126) läßt eine weite Interpretation zu, so daß auch die industriellen Verflechtungen einbegriffen sind. Fünftens führt Marx die Gleichgewichtsbedingung an, unter der die Produzenten stehen. Nur solche Arbeit gilt als gesellschaftlich notwendige, sagt er, die unter den durchschnittlichen gesellschaftlichen Produktionsbedingungen eingesetzt werde. Damit scheiden alle individuellen Sondertatbestände und moralischen Prätentionen für die Preisbestimmung der Waren ebenso aus wie für die Einkommensverteilung (Marx 1867, 53f). Sechstens: Seine Theorie des Konsumentenverhaltens ist denkbar einfach; damit eine Ware überhaupt nachgefragt werden kann, muß sie einen Nutzen für andere haben (Marx 1867, 55). Siebtens: Wie Ricardo so legt auch Marx seiner Preistheorie ein beliebiges, aber gegebenes Niveau der Produktion und Beschäftigung zugrunde. Ganz in der Tradition der klassischen Theorie verbleibend läßt Marx das Preissystem aus den Stromgrößen hervorgehen und stellt es in einen Zusammenhang mit der Reproduktion der Ökonomie. ${ }^{12}$

Diese Gleichgewichtsbetrachtung wird im Mehrwertmodell und im Profitmodell fortgesetzt. Um die Entstehung des Mehrwerts zu erklären, erlegt sich Marx die denkbar härtesten formalen Voraussetzungen auf. Der Mehrwert muß unter der Bedingung erklärt werden, daß die Waren zu ihren Arbeitswerten getauscht werden. Willkürliche Preisaufschläge und andere Sonderbedingungen werden nicht zugelassen. Er muß, wie Marx wiederholt hervorhebt, aus dem »Austausch von Äquivalenten« (Marx 1867, 172f ) entwickelt werden. Schließlich begnügt sich Marx im dritten Band des Kapitals nicht mit der hilflosen Feststellung, daß die Preise von den Arbeitswerten abweichen, sondern er sucht ein Verfahren, um die Preise aller Waren unter der Voraussetzung zu bestimmen, daß es eine allgemeine Profitrate gibt. Marx erkennt, daß die kapitalistische Ökonomie sich nicht re-

12 Marx spricht davon, daß im Austausch der "gesellschaftliche Stoffwechsel" (Marx 1867, 119) stattfinde. Sein Begriff des Formwechsels der Ware bezieht sich hingegen darauf, daß der Wert aus der Warenform in die Geldform übergeht oder umgekehrt. 
produzieren könnte, wenn die Waren zu ihren Arbeitswerten bewertet würden (Marx 1894, 162). Im dritten Band des Kapitals steht er mithin vor dem Problem, daß er ein Gleichgewicht des Kapitalmarktes in Gestalt der allgemeinen Profitrate voraussetzt und ein Gleichgewicht im Gütermarkt sucht, nämlich ein Preissystem, das die Reproduktion der kapitalistischen Ökonomie gewährleistet.

Obgleich Marx auf den historischen Kontext seiner Untersuchung bedacht ist, zeigen seine Gleichgewichtsbegriffe, daß es ihm immer auch um eine strenge Modellbildung für das gesamte System der Märkte geht. Gebührt also auch Marx eine Säule in der Ruhmeshalle der Neoklassiker? Ein gewichtiger Unterschied zwischen Marx und Walras bleibt indes bestehen. Walras setzt voraus, daß die Haushalte über einen gegebenen Anfangsbestand an Gütern verfügen, woraus für die gesamte Ökonomie eine physische Erstausstattung folgt. Weichen die vorhandenen, individuellen Bestände von den präferierten $a b$, dann entsteht aus den individuellen Angeboten und Nachfragen ein Preissystem im Markt, das es jedem Haushalt ermöglicht, durch Tauschakte einen Güterbestand zu erwerben, der mit seinen Präferenzen übereinstimmt. Die walrasianische Tauschtheorie ist eine Allokationstheorie, und ihre Gleichgewichtslösung besteht in einem Preissystem, das die knappen Güter auf die verschiedenen Zwecke in der Reihenfolge ihrer Dringlichkeit verteilt. Alle anderen Probleme: die Produktion, die Kapitalbildung, das wirtschaftliche Wachstum und die Einkommensverteilung werden der Allokationslogik subsumiert. Völlig anders dagegen Marx: Wie bereits oben angeführt, beruht seine Gleichgewichtstheorie auf den Stromgrößen; er nimmt irgendein Aktivitätsniveau an und entwickelt den Austausch als die notwendige Konsequenz einer arbeitsteiligen Ökonomie. Seine Preistheorie ist daher keine Allokationstheorie, sondern eine Reproduktionstheorie. Bei Marx findet sich kein Hinweis auf eine gegebene physische Erstausstattung und sein allgemeines Gleichgewicht geht nicht im neoklassischen Denken auf.

\section{Die industriellen Verflechtungen}

In der modernen ökonomischen Theorie ist es selbstverständlich geworden, die produktiven Beziehungen zwischen den Sektoren als ein interdependentes System zu betrachten, in dem ein Industriezweig sowohl an andere liefert als auch von ihnen Produkte bezieht, die als Produktionsmittel im eigenen Produktionsverfahren eingesetzt werden. Solche Beziehungen zwischen den Sektoren werden als Verflechtungen bezeichnet. Ricardo und die österreichische Schule - zum Beispiel Böhm-Bawerk - haben solchen Interdependenzen keine Aufmerksamkeit geschenkt. Sie argumentieren mit Modellen, die in dieser Hinsicht einfach konstruiert sind: Die Herstellung 
der Konsumgüter wird als ein linearer Produktionsstrom aufgefaßt, der sich über mehrere Produktionsstufen erstreckt. Am Anfang eines solchen Verfahrens wird nur Arbeit eingesetzt, die zusammen mit den Naturkräften das erste Vorprodukt erstellt. Ricardos Beispiel der Tuchproduktion ist das klassische Vorbild eines solchen Fertigungsprozesses (siehe Ricardo 1817, 33f). Ein Unternehmer beschäftigt im ersten Jahr 100 Arbeiter, die Maschinen herstellen, und im zweiten Jahr diesselben 100 Arbeiter, die zusammen mit jenen Maschinen aus der Vorperiode das Konsumgut Tuch erzeugen. Böhm-Bawerk $(1888,11-13)$ übernimmt dieses Produktionsmodell und erweitert es zur Theorie der Produktionsumwege, die ihre eigentliche Bedeutung erst für die neoklassische Kapital- und Zinstheorie erhält. Er begreift jedes Produktionsmittel als ein Konsumgut, das seine Genußreife noch nicht erreicht hat (ebd., 141f). Unter den frühen neoklassischen Ökonomen teilen Jevons und Wicksell diese Ansicht von der gesamtwirtschaftlichen Produktionsstruktur. ${ }^{13}$

Eine gesamtwirtschaftliche Technik, die aus linearen Produktionsströmen zusammengesetzt wird, funktioniert nach dem Vorbild einer Einbahnstraße: Sie kann nur in einer Richtung benutzt werden. Alle Produkte, die unterwegs erzeugt werden, dienen stets nur den nachfolgenden Fertigungsstufen als Produktivgut. Marxens Untersuchung der gesamtwirtschaftlichen Produktionsbeziehungen ist nicht auf eine solche Technik beschränkt, sondern schließt ein Produktionsmodell ein, das aus den interdependenten Produktionsströmen hervorgeht und es gestattet, die Kreislaufverflechtungen der Ökonomie in die Darstellung aufzunehmen. Schon im Modell der einfachen Warenzirkulation spricht er von der »allseitigen sachlichen Abhängigkeit « der Produzenten ${ }^{14}$ und beschreibt eine industrielle Produktion mit vielfältigen Vorleistungsverflechtungen. Überdies kann auch der Begriff der »gesellschaftlich notwendigen Arbeit« als Ausdruck für die industriellen Verflechtungen verstanden werden, wie Morishima ausführt, weil der Arbeitswert einer Ware alle direkt und indirekt in der Ökonomie aufgewendeten Arbeitsmengen enthält, die zu ihrer Herstellung nötig waren. ${ }^{15}$ Eine gründliche Untersuchung der Kreislaufbeziehungen findet sich hier jedoch ebensowenig wie im Profitmodell. ${ }^{16}$

Am Ende des zweiten Bandes des Kapital, noch im Rahmen seiner Mehrwerttheorie, entwickelt Marx ein formales Modell der gesamtwirtschaftli-

13 Siehe Jevons $(1871,224-229)$ und Wicksell $(1913,213$ f sowie sein Beipiel der weinproduzierenden Ökonomie 238ff).

14 Marx (1867, 122); auch Produktionsmittel gehen in diese Zirkulation ein (ebd., 120).

15 Morishima $(1973,11 \mathrm{f})$ denkt dabei an eine Darstellung der Marxschen Arbeitswerte in einer Leontief-Tafel der Input-Output-Beziehungen.

16 Siehe Marx (1894, 165f). Ob Marx bei der Untersuchung der Produktionspreise ein ökonomisches System mit linearen oder interdependenten Produktionsströmen vor Augen hatte, kann aus dem Text nicht geschlossen werden. 
chen Reproduktion, in dem die Industriezweige durch Vorleistungsverflechtungen miteinander verbunden sind (Marx 1885, Kapitel 20 und 21). Im folgenden vereinfachen wir das Marxsche Modell der einfachen Reproduktion: wir setzen eine stationäre Ökonomie voraus, in der es nur Vorprodukte als Produktionsmittel gibt, aber kein fixes Kapital; die Arbeit sei homogen und vom Boden werde abgesehen. Die beiden gesamtwirtschaftlichen Produktionssektoren, von denen Marx (1885, 394-401) spricht, nämlich die Produktivgüterindustrien und die Konsumgüterindustrien, reduzieren wir auf zwei Industriezweige, in denen jeweils ein Gut hergestellt wird. Im Unterschied zu Marx verzichten wir auf die Arbeitswertrechnung und geben die Tauschrelationen der Waren zu Produktionspreisen an.

In dieser Analyse erreicht Marx einen bedeutenden Fortschritt. Zum einen betrachtet er die Industriezweige in ihrem Zusammenhang miteinander und bringt die Austauschbeziehungen, die zwischen ihnen bestehen, in eine geschlossene Form. Zum anderen stellt er erstmalig ein Produktionsverfahren formal dar, in dem ein Produkt zugleich als Produktionsmittel in seinem eigenen Herstellungsprozeß verwendet wird; dies ist der einfachste Fall einer industriellen Verflechtung. In Marxens Modell wird das Produktionsmittel - nennen wir es Maschine - auf folgende Weise hergestellt: Maschinen werden durch den Einsatz von Maschinen und Arbeit produziert. In einem zweiten Produktionszweig wird das Konsumgut - im folgenden als Tuch bezeichnet - erzeugt, indem wiederum Arbeit und Maschinen benutzt werden. Das Tuch dient allein dem privaten Verbrauch und geht nicht in die Produktion ein. Die gesamtwirtschaftliche Produktionstechnik kann formal durch die Beziehungen:

$$
\begin{array}{ll}
\mathrm{x}_{1}+\mathrm{L}_{1} \rightarrow \mathrm{x} & \text { 1. Industrie: Produktivgut Maschine } \\
\mathrm{x}_{2}+\mathrm{L}_{2}-\mathrm{y} & \text { 2. Industrie: Konsumgut Tuch }
\end{array}
$$

dargestellt werden, wobei die Ausbringungsmenge des Produktionsmittels Maschine mit $\mathrm{x}$ und die des Konsumgutes mit y bezeichnet wird; das Symbol $\mathrm{x}_{1}$ gibt die Einsatzmenge der Maschinen in der Maschinenindustrie und das Symbol $x_{2}$ ihre Einsatzmenge in der Tuchindustrie an; ganz ähnlich bedeuten $\mathrm{L}_{1}$ und $\mathrm{L}_{2}$ die Einsatzmengen der Arbeit in den jeweiligen Zweigen. Die Produktionskosten in dieser Ökonomie können in zwei Kostengleichungen dargestellt werden, in denen $r$ für die Profitrate, w für den Nominallohnsatz sowie $\mathrm{p}$ und $\mathrm{q}$ für die Preise der entsprechenden Güter stehen. Man erhält: ${ }^{17}$

$$
\left(\mathrm{px}_{1}+\mathrm{w} \mathrm{L}_{1}\right)(1+\mathrm{r})=\mathrm{px}
$$

17 Gleichungen für die Produktionspreise, in denen konstante Produktionskoeffizienten enthalten sind, finden sich bereits bei Walras $(1874,240)$. 


$$
\left(\mathrm{px}_{2}+\mathrm{w} \mathrm{L}_{2}\right)(1+\mathrm{r})=\mathrm{q} \mathrm{y}
$$

Unter der Bedingung, daß sich die Ökonomie gerade reproduzieren kann und daher alles verbraucht, was sie erzeugt, gelten die folgenden Gleichungen:

$$
\begin{aligned}
& (1+\mathrm{r}) \mathrm{w}\left(\mathrm{L}_{1}+\mathrm{L}_{2}\right)+\mathrm{r}\left(\mathrm{px}_{1}+\mathrm{px}_{2}\right)=\mathrm{qy} \\
& \mathrm{p}\left(\mathrm{x}_{1}+\mathrm{x}_{2}\right)=\mathrm{px}
\end{aligned}
$$

Die erste dieser beiden Gleichungen besagt, daß das gesamte Einkommen, bestehend aus Arbeitslohn und Profit, für den Kauf des Konsumgutes Tuch verwendet wird; aus der zweiten ersieht man, daß die gesamte Ausbringungsmenge an Maschinen benutzt wird, um die verbrauchten Produktionsmittel zu ersetzen. Aus der Kostengleichung [2] und der Bedingung [3] für die einfache Reproduktion folgt Marxens Fundamentalgleichung für eine stationäre Ökonomie: ${ }^{18}$

$$
\mathrm{w} \mathrm{L}_{1}+\mathrm{r}\left(\mathrm{w} \mathrm{L}_{1}+\mathrm{px}_{1}\right)=\mathrm{px}_{2}
$$

Die letzte Gleichung gibt die Verflechtungen zwischen den beiden Industrien wieder, wenn die Ökonomie sich auf einem gleichbleibenden Niveau reproduziert. Der Austausch zwischen den beiden Industriezweigen muß also zwei Bedingungen genügen, damit das ökonomische System sich tatsächlich reproduzieren kann. Erstens, indem die Konsumgüterindustrie eine bestimmte Menge Konsumgüter, deren Wert $\left(\mathrm{px}_{2}\right)$ beträgt, an die Produktivgüterindustrie verkauft, ersetzt sie ihre verbrauchten Produktionsmittel. Umgekehrt werden die in der Produktivgüterindustrie entstandenen Einkommen: der Lohn $\left(\mathrm{wL}_{1}\right)$ und der Profit $\left[\mathrm{r}\left(\mathrm{wL}_{1}+\mathrm{px}_{1}\right)\right]$ durch diesen Austausch in Konsumgüter verwandelt. Zum zweiten müssen diese Transaktionen zu solchen Preisen vonstatten gehen, daß jeder Produktionssektor den Durchschnittsprofit realisieren kann.

Mit dieser Untersuchung hat Marx die Grundlagen für die Analyse der Kreislaufverflechtungen gelegt. Leontief (1938, 3-5) und Samuelson (1974, 270-272) erkennen in den Reproduktionsmodellen seine eigentliche Leistung für die ökonomische Analyse. Marx trägt mit diesen Untersuchungen einen Baustein zur Input-Output-Analyse bei, die von Leontief in den 30er und 40er Jahren entwickelt wurde. ${ }^{19}$

Gerade diese Besonderheit weist das Ricardo-Böhm-Bawerk-Modell nicht

18 Siehe Marx $(1885,401)$. In der Marxschen Notation lautet diese Beziehung: $\mathrm{cII}=\mathrm{vI}+$ mI. Die Indizes I und II bezeichnen die Produktivgüter- und die Konsumgüterindustrie, c gibt das konstante Kapital, v das variable Kapital und m den Mehrwert an; alle Größen werden in Arbeitswerten gemessen. - Die Bedingung für die einfache Reproduktion kann auch aus den Gleichungen [1] und [4] abgeleitet werden.

19 Siehe auch Samuelson (1957, 888f). Sraffa (1960), John v. Neumann (1945/46) und Walras haben ebenfalls solche industriellen Verflechtungen in ihre Modelle integriert. 
auf. Sehen wir hier einmal davon ab, daß die Arbeit für Ricardo eine produzierte Ware und für Böhm-Bawerk eine exogene Größe und daher eine Ressource ist, dann kann das Herstellungsverfahren für das Konsumgut Tuch durch die Beziehungen:

$$
\begin{array}{ll}
\mathrm{L}_{1} \rightarrow \mathrm{x} & \text { 1. Stufe: Produktivgut Maschine } \\
\mathrm{x}_{2}+\mathrm{L}_{2}-\mathrm{y} & \text { 2. Stufe: Konsumgut Tuch }
\end{array}
$$

formal dargestellt werden. Aus diesen produktiven Verknüpfungen erhält man die Kostengleichungen

$$
\begin{aligned}
& \mathrm{wL}_{1}(1+\mathrm{r})=\mathrm{px} \\
& \left(\mathrm{p} \mathrm{x}_{2}+\mathrm{w}_{2}\right)(1+\mathrm{r})=\mathrm{q} \mathrm{y},
\end{aligned}
$$

wobei wir auch in diesem ricardianisch-österreichischen Modell einen stationären Zustand annehmen, ohne dies jedoch formal auszuführen.

Bislang haben wir das Marxsche Reproduktionsmodell unter der stark vereinfachenden Annahme betrachtet, daß in der Ökonomie nur zwei Waren produziert werden. Unter dieser ausgesprochen rigiden Voraussetzung verschwindet eine der Stärken der Marxschen Analyse. Wie Leontief (1936, 4) hervorhebt, weist das Reproduktionsmodell den Vorzug auf, daß eine aus vielen Produktionszweigen zusammengesetzte Ökonomie auf zwei Sektoren zurückgeführt wird, die im Hinblick auf ihre funktionale Bedeutung für die Gesamtwirtschaft gegeneinander abgegrenzt werden. Marxens Modell sei anderen überlegen, urteilt Leontief, weil es die wesentlichen Merkmale moderner ökonomischer Systeme zum Ausdruck bringe. Zum einen leiste seine Betrachtungsweise mehr als das Modell der linearen Produktionsströme von Böhm-Bawerk, das einfach unrealistisch sei, und zum anderen mehr als der Ansatz von Walras, der die Verflechtungen zwischen den Einzelwirtschaften in einem höchst disaggregierten Modell aufnehme, das daher für Zwecke der empirischen Untersuchung nicht handhabbar sei. ${ }^{20}$

\section{Produktionsstruktur, Preisbestimmung und Verteilung}

Ricardo, Marx und Walras benutzen Produktionsmodelle, die stark voneinander abweichen und in je verschiedenen Beziehungen zur Preis- und Verteilungstheorie stehen. Ricardo arbeitet mit Hilfe der linearen Produktionsströme den Einfluß heraus, den Verteilungsänderungen auf das Preissystem haben. Bei Marx wird die Theorie der Produktionspreise noch getrennt von

20 Walras $(1874,239 f, 271)$ berücksichtigt in seinem Modell die industriellen Verflechtungen zwischen den Industriezweigen und fügt sie in seine Theorie der Preisbestimmung ein. - Ähnlich wie Leontief argumentierte schon Nurkse (1934/35, 241f), der für eine Theorie der Konjunkturschwankungen eine wirklichkeitsnahe Beschreibung der Produktionsstruktur forderte und sich mit dem Modell der linearen Produktionsströme nicht zufrieden gab. 
seinem aggregierten Reproduktionsmodell behandelt, und Walras schließlich verknüpft ein disaggregiertes Modell der Kreislaufverflechtungen mit der Preistheorie. Beide - sowohl Marx wie Walras - schenken dem Einfluß der Verteilungsänderungen auf die relativen Preise keine Aufmerksamkeit. ${ }^{21}$ In den Theorien von Ricardo und - mit noch größerem Gewicht von Marx kommt der Verteilung des Einkommens auf Lohn und Profit eine entscheidende Bedeutung zu, während Walras diese Frage eher als eine Nebensache zu behandeln scheint.

Das Modell der linearen Produktionsströme, das durch Ricardos Arbeit berühmt geworden ist, hat in der Geschichte der Preistheorie nachgewirkt. Wicksell arbeitete mit einem solchen Modell und der russische Wirtschaftstheoretiker Dmitriev benutzte es, um die Kritik von Walras an der Werttheorie Ricardos zurückzuweisen. Walras $(1874,424 f)$ hatte gegen Ricardo den Einwand erhoben, daß dessen ökonomisches System nicht determiniert sei, weil es - in formaler Betrachtung - über mehr Variable als Gleichungen verfüge. Dmitriev $(1898,87-92)$ konnte als erster in einer formal geschlossenen Darstellung zeigen, daß Ricardos Preis- und Verteilungstheorie determiniert und Walras' Kritik unberechtigt war. Um die linearen Produktionsströme mit der Preistheorie zu verbinden, werden die auf den einzelnen Produktionsstufen auftretenden Arbeitsmengen datiert und mit dem Lohnsatz bewertet. Im allgemeinen wird das Verhältnis der Güterpreise nicht dem Verhältnis der Arbeitsmengen gleichen, weil ein Zinseszinseffekt auftritt. Bortkiewicz (1907a, 29 und 33) greift auf die Lösung von Dmitriev zurück und benutzt das Konzept der datierten Arbeitsmengen, um zu zeigen, daß auch die Marxsche Preis- und Verteilungstheorie determiniert ist.

In einem weiteren Aufsatz entwickelt Bortkiewicz (1907b, 321) eine Lösung für das Preissystem, indem er zwei Argumentationen miteinander verknüpft, nämlich die Theorie der Produktionspreise und das einfache Reproduktionsmodell, die in der Marxschen Theorie noch voneinander getrennt sind. Dieser Beitrag ist der erste gelungene Versuch, die klassische Werttheorie mit den industriellen Verflechtungen zu verbinden. Leontief nun, der bei Bortkiewicz promovierte, entwickelt mit der Input-OutputAnalyse und der linearen Produktionstheorie formalisierte Verfahren zur Untersuchung der gesamtwirtschaftlichen Kreislaufzusammenhänge, die in den 50er Jahren aus der empirischen Wirtschaftsforschung - ihrem eigentlichen Anwendungsgebiet - auf theoretische Fragestellungen übertragen werden. Zu Beginn der 50er Jahre zeigt May (1949/50, 61 und 65f) mit Hilfe der linearen Produktionstheorie, daß die Marxschen Produktionspreise

21 Marx $(1894,210-14)$ nimmt diese Frage zwar auf, aber es handelt sich dort weniger um eine eigenständige Untersuchung als um eine Paraphrase von Ricardos Ausführungen. 
auf ein allgemeines Gleichgewicht führen; dabei verweist er auf die Ähnlichkeit dieses Gleichungssystems mit den Modellen von Walras und Leontief, ohne jedoch auf die industriellen Verflechtungen näher einzugehen. Samuelson $(1957$; 1959) benutzt diese neuen Instrumente, um die klassische Werttheorie zu reformulieren. Seton (1956/57) führt die Input-OutputAnalyse in die Marxsche Arbeitswerttheorie ein, um die Produktionspreise und die allgemeine Profitrate zu bestimmen. ${ }^{22}$ Während sein historisches Vorbild Bortkiewicz sehr eng am Marxschen Reproduktionsmodell bleibt, ist das Modell von Seton vollkommen allgemeingültig und enthält eine beliebige Anzahl von Industriezweigen. Damit gelingt ihm die erste formale Darstellung der Marxschen Preistheorie, in der drei Stränge des ökonomischen Denkens zusammengeführt werden, die bis dahin in den Diskussionen um die Marxsche Theorie getrennt verliefen: er verknüpft in einem allgemeinen, disaggregierten Gleichgewichtsmodell die industriellen Interdependenzen mit der Preisbestimmung. Zwei Dinge werden im Rahmen dieser Analyse deutlich: erstens, wenn Bortkiewicz und Seton von den aufgespeicherten Arbeitsmengen ausgehen, um die Produktionspreise $\mathrm{zu}$ bestimmen, dann gelingt ihnen dieser Versuch deshalb, weil sie ein Rechenverfahren benutzen, mit dessen Hilfe die in den Arbeitsmengen verborgene Produktionstechnik dechiffriert werden kann. Mit diesen Informationen ausgestattet können sie das Preissystem und die Profitrate determinieren, wenn der Reallohnsatz bekannt ist. Zweitens ersieht man daraus, daß die Produktionstechnik, nicht aber die Arbeitsmengen notwendig sind, um die Unbekannten zu ermitteln. ${ }^{23}$ Die Marxschen Arbeitswerte sind in dieser Sichtweise nichts anderes als eine etwas umständliche Art, die Produktionsmethode der gesamten Ökonomie zu beschreiben - und da dies die einzigen Informationen sind, welche das Marxsche Modell über die Technik bereithält, bilden sie den Ausgangspunkt der Preisbestimmung. Auf diese Weise benötigt man im Grunde nur einen Rechenschritt mehr, um das angestrebte Ziel zu erreichen. ${ }^{24}$

Die Kontroversen um die Marxsche Verteilungstheorie wurden von dem Problem beherrscht, die Exploitation der Arbeiter zu begründen, indem die Mehrarbeit gewissermaßen als Substanz des Profits nachzuweisen war. ${ }^{25}$

22 Ebenfalls in den frühen 50er Jahren stellt Cameron (1952) eine Beziehung zwischen der Marxschen Werttheorie und dem Modell Leontiefs her. Sobald nur ein originärer Produktionsfaktor - die Arbeit - existiert, entsprechen die Preise des Leontief-Modells den Marxschen Arbeitswerten. Dieses Ergebnis ist weniger um seiner selbst willen erstaunlich, sondern weil es zeigt, daß der Sonderfall der klassischen Werttheorie unversehens in einem ganz anderen theoretischen Kontext auftritt.

23 Siehe dazu Seton (1956/57, 151 Fn. 3).

24 Samuelson $(1957,911)$ spricht von einer 'unnecessary detour' und Steedman $(1977,202)$ hält die Arbeitswerttheorie für redundant.

25 Vgl. Sweezy $(1942,156)$ und Meek (1956, 181, 194ff), wo Meek von einer gelungenen 
Diese Debatten scheinen die Auseinandersetzung mit der klassischen Werttheorie und der Marxschen Theorie im Besonderen aber eher gehemmt als gefördert zu haben, weil sie stets um das Problem kreiste, ob die Warenpreise in irgendeiner Beziehung zu den aufgewendeten Arbeitsmengen stünden. Diese Verengung der Diskussion um die klassische Verteilungstheorie wird sehr spät überwunden. Erst in Sraffas Schrift Warenproduktion mittels Waren aus dem Jahre 1960 wird das in Vergessenheit geratene Problem Ricardos aufgegriffen und das allgemeine Gleichgewichtsmodell um die gewichtige Frage erweitert, wie ein höherer oder niedrigerer Reallohnsatz auf die relativen Preise wirkt. Damit wirft Sraffa ein neues Licht auf die Preistheorie: Er verbindet die Idee der Kreislaufverflechtungen, wie sie bei Marx und Walras vorhanden ist und im Rahmen der Input-OutputAnalyse ausgearbeitet wird, mit dem Problem, wie Verteilungsänderungen auf die relativen Preise wirken, das Ricardo behandelt, in einem allgemeinen, disaggregierten Gleichgewichtsmodell. Diese Untersuchung führt zur Einsicht in die verteilungsinduzierten Umbewertungen, die in jedem allgemeinen Gleichgewichtsmodell enthalten sind, ob dieses nun der klassischen Tradition von Ricardo und Marx oder der neoklassischen Tradition von Walras entstammt: wenn die Verteilungsgrößen wechseln, bleibt das System der relativen Preise davon nicht unberührt. Rückblickend zeigt sich, daß Sraffa die beiden großen Fragestellungen, welche die Begründer der modernen ökonomischen Theorie im 19. Jahrhundert untersuchten: die Einkommensverteilung und die Preisbestimmung, in einer Gleichgewichtstheorie zusammenfaßt, wobei er die klassische Werttheorie reformuliert und von ihrer Bindung an die Arbeitsmengen befreit und in das Walrasianische System eine Fragestellung einführt, die Walras selbst nicht interessierte. In umgekehrter Richtung führt er die Produktionstechnik, die Walras in den Produktionskoeffizienten erfaßt, ohne Umwege über aufgespeicherte Arbeitsmengen in die Gleichungen der klassischen Preistheorie ein.

Wir können Sraffas Modell benutzen, um das zuvor angeführte Marxsche Preissystem zu determinieren. In den Gleichungen [1] und [2] gibt es zwei Güterpreise, den Lohnsatz und die Profitrate, zusammen also vier Unbekannte. Fügt man eine dritte Gleichung hinzu: $w=q c_{y}$, die den Subsistenzlohn der Arbeiter enthält, wobei die Konstante $c_{\mathrm{y}}$ die Menge des notwendigen Lebensmittels Tuch angibt, und eine weitere, vierte Gleichung, die den Wertstandard festlegt, in dem alle Preise und der Lohnsatz w gemessen werden: $q=1$, dann sind der Maschinenpreis und die Profitra-

Transformation der Arbeitswerte in Preise ausgeht. Später wird er allerdings deutlich vorsichtiger. So schreibt er im Vorwort zur zweiten Auflage seiner Studies, daß das Modell Sraffas den gleichen Zweck erfülle wie die Marxsche Arbeitswerttheorie: Um die Preise zu bestimmen, müsse die Produktionstechnik bekannt sein. Somit stelle sich die Frage nach der Relevanz der Arbeitswerttheorie (Meek 1956, XXIII, XLII; Meek 1977, 119). 
te ermittelt und das Marxsche Modell ist determiniert.

\section{Geld und die Rentabilität von Kapitalvorschüssen}

Keynes wertete Marxens 'allgemeine Formel des Kapitals': G - W - G' als eine bemerkenswerte Leistung, die für dessen Theoriebildung jedoch ohne Konsequenzen geblieben sei. Diese Formel birgt in der Tat ein spezifisches Verständnis gesamtwirtschaftlicher Zusammenhänge: Geld wird vorgeschossen, um Produktionsprozesse durchzuführen, und das Produktionsergebnis ist nicht als physische Gütermenge von Interesse, sondern als monetäre Größe, genauer: als Überschuß des Geldrückflusses über den Geldvorschuß. In der kapitalistischen Produktion, so Marx $(1894,51)$, wird der Profit als ein monetärer Überschuß angestrebt.

Mit dem vierten Kapitel im ersten Band des Kapital nimmt die Marxsche Theorie eine überraschende Wende. Bis dahin beruht die Argumentation auf Stromgrößen, aus denen das System der relativen Preise entwickelt wird. Sobald Marx jedoch den Begriff des Kapitals aufnimmt, geht er von einer monetären Bestandsgröße aus: ein Geldvermögensbestand wird nun den Stromgrößen: der Produktion und dem Einkommen vorausgesetzt. An die Stelle eines beliebigen Produktionsvolumens tritt ein Geldvermögensbestand, dessen Höhe bei gegebener Technik, einer bekannten Profitrate und gegebenen Preisen das Aktivitätsniveau der Ökonomie determiniert. ${ }^{26}$ Hier beginnt eine neue Theorie: Sie unterscheidet sich von der klassischen Theorie Ricardos, weil Marx die Untersuchung der kapitalistischen Produktion nicht mit einer vorausgesetzten Stromgröße, sondern mit einem gegebenen Vermögensbestand eröffnet. An die Stelle der Reproduktion physischer Gütermengen tritt die Erhaltung und Vermehrung des Geldvermögensbestandes. Auf der anderen Seite teilt sie zwar mit der allgemeinen Gleichgewichtstheorie von Walras den Ausgangspunkt in einer Bestandsgröße, setzt aber anders als diese keinen physischen Güterbestand, sondern eben ein Geldvermögen voraus.

Sowohl von Ricardo als auch von Walras unterscheidet sich Marx durch die Rolle, die dem Geld in der Ökonomie zugewiesen wird. Obgleich er letzteres aus dem Austausch der Waren ableitet, wäre es falsch, die Funktion des Geldes in seiner Theorie auf die eines Wertstandards zu reduzieren. Vielmehr zeigt die einfache Warenzirkulation, daß Marxens Arbeitswerttheorie neben einer technischen Arbeitsmengentheorie der Preise eine zweite Schicht beinhaltet, in der die Begriffe Arbeit, Austausch und Geld einen gesellschaftstheoretischen Gehalt gewinnen: Marxens Theorie des Warenaustausches begreift sowohl die sozialen Voraussetzungen ein, wel-

26 Wir setzen konstante Skalenerträge voraus. Ferner wird eine Arbeiterbevölkerung angenommen, deren Größe ausreicht, um dieses Produktionsvolumen zu erzeugen. 
che die Einzelwirtschaften zum Austausch zwingen, als auch die sozialen Bedingungen, unter denen sich die Koordination der vielen einzelnen Produzenten vollzieht. Mit dem Begriff der »Privatarbeit« bezeichnet Marx diejenigen Verhältnisse, in denen die Produktion in einem System der gesellschaftlichen Arbeitsteilung für den Markt stattfindet, und stellt ihm die Kategorien Ware und Geld gegenüber; der Ware und dem Geld fällt die Aufgabe zu, die Produzenten innerhalb dieser arbeitsteiligen Ökonomie zu integrieren. Der Austausch zwischen Ware und Geld wird zu der entscheidenden sozialen Beziehung, mittels derer die Gesellschaft sich reproduziert. In der Marxschen Tauschtheorie stellt das Geld eine tatsächliche soziale Beziehung zwischen handelnden »Warenbesitzern« her.

Möglicherweise hat Keynes mit seinem Verdikt über die »allgemeine Formel des Kapitals « recht: Marx untersuchte zwar ausführlich die Funktionen des Geldes im Austausch, aber dessen Bedeutung als Vermögensbestand für die Durchführung von Produktionsprozessen wurde wenig Aufmerksamkeit gewidmet. Marx beläßt es bei den Ausführungen im vierten Kapitel des ersten Bandes vom Kapital und kommt, von einigen Bemerkungen im zweiten Band abgesehen, erst im dritten Band bei der Betrachtung des Kredits wieder darauf zurück. Das Geldvermögen wird vom Gläubiger auf den Schuldner übertragen, der nun seinerseits - in der Rolle des Unternehmers - das Kapital für den Kauf der Produktionsmittel und zur Entlohnung der Arbeitskraft vorschießt; der doppelte Vorschuß des Geldvermögensbestandes zieht einen doppelten Rückfluß nach sich (Marx 1894, 352f). Jetzt hängen die Produktion und die Beschäftigung von einem doppelten Vorschuß des Geldvermögens ab, und der gesamte Prozeß ist erst dann abgeschlossen, wenn die Hauptsumme des Kapitals zusammen mit dem Zins auf den Eigentümer rückübertragen wird. Marxens Untersuchung zeigt, daß die Produktions- und Einkommensströme in ein weitgespanntes Netz von monetären Vermögensbeständen und ihren Übertragungen eingespannt sein können. Indes bleiben seine Ausführungen zum Zusammenhang des Geldes mit dem Kredit sowie die Beziehungen zwischen der Zentralbank und den Geschäftsbanken fragmentarisch; die Frage, woher das Geld kommt, das in den Kreditverträgen übertragen wird, und die weitere, welche Gesetze den Geldumlauf unter dem Kreditsystem regulieren, bleiben letztlich ungeklärt. Kehrt man zur allgemeinen Formel des Kapitals zurück und betrachtet deren Implikationen genauer, so wird deutlich, daß sie bereits eine Definition der allgemeinen Profitrate enthält, obgleich Marx erst mehr als eintausend Seiten später ausführlich auf die letztere eingehen wird. Bezieht man den monetären Überschuß $\Delta \mathrm{G}$, der in dieser Formel ausgewiesen wird, auf den Geldvorschuß G, so erhält man die Rate: $r=\Delta \mathrm{G} / \mathrm{G}$, die als monetäre Profit- 
rate verstanden werden kann. ${ }^{27}$ Seine Kreislaufformel des Kapitals verknüpft also Bestandsgrößen mit Stromgrößen und enthält den Ansatzpunkt einer Verteilungstheorie. Die monetäre Profitrate weist eine gewisse Ähnlichkeit mit der monetär bestimmten Zinsrate auf, die in der Keynesschen Theorie eine herausgehobene Rolle spielt. Bei Marx ist der monetäre Profit nur durch einen vorangehenden Geldvorschuß zu erlangen, und bei Keynes $(1936,140)$ tritt der Zins als Belohnung für die Aufgabe von Liquidität auf. Beiden ist somit die monetäre Bestimmung des Profits respektive des Zinses gemeinsam; sieht man vom Unternehmergewinn ab, der im Gleichgewicht Null wird, so sind Profit und Zins das Gleiche. Diese monetären Rentabilitätsraten unterscheiden gleichermaßen Marx und Keynes von jenen Vertretern der Neoklassik, welche die allgemeine Profitrate aus der Grenzproduktivität einer Menge abzuleiten suchen.

Eine weitere Parallele zwischen Marx und Keynes zeigt sich bei der Bestimmung des Aktivitätsniveaus der Ökonomie. Wie bereits Joan Robinson (1942, 73f) feststellte, hatten weder Keynes noch Marx ein Investitionskalkül für einen weit in die Zukuft reichenden Zeitraum entwickelt. Marx neigt dazu, ein Zwangsgesetz der Akkumulation anzunehmen, welches die Unternehmer zu immer neuen Investitionen treibt, während das langfristige Niveau der Produktion bei Keynes eine Angelegenheit der animal spirits der Lebensgeister - ist (Keynes 1936, 136 und 316). Darin liege jedoch kein Mangel, so Joan Robinson weiter, weil ein Investitionskalkül für die langfristige Analyse ohnehin unbedeutend sei. Es gibt keine langfristige Beziehung zwischen dem Aktivitätsniveau einer Ökonomie und der Renditerate: $\gg . .$. any prospective level of profit, within very wide limits, is sufficient to keep the system running « (Robinson 1942, 73). Bei der kurzfristigen Analyse hält Marx die Antwort auf die Frage, wodurch die Höhe der Geldvorschüsse bestimmt werde, in der Schwebe. ${ }^{28}$ Ein wirkliches Investitionskalkül, wie es Keynes mit der Veranlassung zur Investition formuliert, fehlt ihm.

Beiden Autoren gemeinsam ist auch die Verbindung der Einkommensverteilung mit dem Aktivitätsniveau. In der Marxschen Akkumulationstheorie (Marx 1967, 648ff) hängt die Wachtumsrate des Kapitalbestandes mit dem gegensätzlichen Verhältnis zwischen der Mehrwertrate und dem Reallohnsatz zusammen; sinkt die Mehrwertrate, dann stagniert die Akkumulation.

27 Siehe auch Marx (1863-67, 52f). Dort wird diese Rate der Sache nach - anhand eines numerischen Beispiels - eingeführt. Im dritten Band des Kapital definiert Marx $(1894,52)$ indes die Profitrate als ein Verhältnis zwischen aufgespeicherten Arbeitsmengen, und eine solche Rate ist im allgemeinen von der tatsächlichen Profitrate des ökonomischen Systems verschieden. Diese aus der Arbeitswertrechnung stammende Rate gibt Marx fehlerhafter Weise für die allgemeine Profitrate aus.

28 Marx $(1894,529)$ erwähnt zwar eine erwartete Profitrate, aber sie bleibt für seine Argumentation ohne Bedeutung und steht ganz im Zusammenhang mit der Spekulation. 
Die Idee schließlich, daß die Profitrate langfristig fallen werde, schließt eine inverse Beziehung zwischen der Profitrate und der Höhe des Kapitalbestandes ein. Auch Keynes stellt eine solche Beziehung her. Die Kapitalvorschüsse werden demnach knapp gehalten, weil die erwartete Profitrate zumindest gleich der Zinsrate auf Geld sein muß (Keynes 1936, 178f). Folglich fällt bei einem gegebenen Stand der Erwartungen das Aktivitätsniveau um so geringer aus, je höher die Zinsrate ist.

Beide Theorien vermuten einen Zusammenhang zwischen Verteilungsänderungen und den Mengeneffekten im Gütermarkt und im Arbeitsmarkt. Einem solchen Kausalzusammenhang stehen allerdings die Verwicklungen entgegen, welche die Kapitaltheorie bereithält.

\section{Die Kapitaltheorie und das Aggregationsproblem}

Wie der Untertitel des Kapital ankündigt, geht es in diesem Buch um eine »Kritik der politischen Ökonomie« und das heißt bei Marx, vor allem um eine Kritik der klassischen ökonomischen Theorien von Adam Smith und David Ricardo. Soweit man jene Teile seiner Arbeit betrachtet, die Morishima als einen Beitrag zur allgemeinen Gleichgewichtstheorie wertet, zeigt sich Marx als ein normaler Wirtschaftstheoretiker: Seine Preistheorie und seine Verteilungstheorie enthalten nichts Revolutionäres, sondern sie setzen die Tradition fort, die von Ricardo begründet wurde. Aber innerhalb dieses überlieferten Rahmens entwickelt er eine grundsätzliche Kritik des ökonomischen Denkens und eine ökonomisch fundierte Kritik der bürgerlichen Gesellschaft; er verbindet die Existenz von Klassen mit einer Exploitationstheorie, derzufolge die 'Aneignung unbezahlter Mehrarbeit' ein normales Merkmal der kapitalistischen Ökonomie sei. Vielleicht stärker als alles andere im Kapital barg die Exploitationstheorie einen frontalen Angriff auf das liberale ökonomische Denken und auf eine Vorstellungswelt, in der jedes Einkommen auf individuelle Leistungen zurückgeführt und daher als gerecht empfunden werden konnte.

In der Produktionsfaktorentheorie fand diese liberale Auffassung einen Niederschlag; im Umriß war sie bereits bei Adam Smith vorhanden; sie wurde von Marshall - um eine ausgewogene Position stets bemüht - aufgenommen und ergänzt. Und bei J.B. Clark rückte das Bestreben nach sozialer Rechtfertigung des Profits so aufdringlich in den Vordergrund, weil bei ihm im Unterschied zu anderen zeitgenössischen Autoren wie Wicksell alle wissenschaftlichen Bedenken gegen die Aggregation heterogener physischer Güter zu einer homogenen Größe verschwunden waren. ${ }^{29}$ Wie dem auch sei - jenseits aller ideologischen Kontroversen richtete sich die Anti-

29 Siehe schon Smith (1776, 69), Marshall (1890, 62 und 66-69) und Clark (1899, 2-10). 
kritik auf das eigentliche ökonomische Problem, nämlich auf den Erklärungszusammenhang, den Marx zwischen den Arbeitswerten, der Mehrarbeit und dem Profit entwickelt hatte; es wurde gezeigt, daß die Preise nicht durch die Arbeitswerte reguliert werden. Am Transformationsproblem der Frage also, ob die Arbeitswerte in Gleichgewichtspreise verwandelt werden können - wurde erfolgreich gezeigt, daß, erstens, das Verhältnis der Preise nicht mehr gleich dem Verhältnis der Arbeitswerte ist; diese Schwierigkeit hatte Marx durchaus gesehen, aber im weiteren beiseite geschoben (siehe Marx 1894, 162). Dies konnte ihm um so leichter fallen, als er der Überzeugung war, daß die gesamtwirtschaftliche Summe der Arbeitswerte gleich der Summe der Produktionspreise und der gesamte Mehrwert gleich dem gesamten Profit wäre. Wie - zweitens - die Diskussion ergab, ist diese Annahme falsch. ${ }^{30}$ Der Grund dafür liegt im Einfluß der Einkommensverteilung auf die Bestimmung der Preise; diesen Zusammenhang erkannte zuerst Ricardo $(1817,35)$ und bezeichnete die verteilungsinduzierten Umbewertungen der Waren als curious effect (Ricardo 181618, 82). Anders als Ricardo quälte Marx sich mit dem Versuch, das System der Arbeitswerte mit demjenigen der Produktionspreise zu versöhnen. ${ }^{31}$ Der Kardinalfehler - wenn man so will - der Marxschen Preistheorie besteht darin, daß Marx eine aus der Arbeitswertrechnung gewonnene Rate als allgemeine Profitrate mißversteht und sie benutzt, um den Mehrwert proportional zum Kapitalwert, der in Arbeitswerten gemessen wird, auf die Industriezweige zu verteilen. Auf diese Weise wird ein gegebener Mehrwert auf die vorhandenen Industrien umverteilt, und das Ergebnis nennt er Durchschnittsprofit. Es ist jedoch so, daß nicht nur die Absatzpreise der Güter, sondern auch die Preise der Einsatzmengen von den Arbeitswerten auf die Produktionspreise umgestellt werden müssen. ${ }^{32}$ Marx übersieht, daß der Kapitalwert und die Einkommensverteilung voneinander abhängen. Mit diesem Fehler steht er jedoch nicht allein!

Das Transformationsproblem ist tatsächlich nur der besondere Fall des Aggregationssproblems, das eben nicht nur der klassischen Werttheorie, sondern jeder Preistheorie eigen ist und das immer dann auftritt, wenn die Wertgröße heterogener Güterbündel ermittelt werden muß. Wenn die Rechnung lautet: Preise mal Mengen, dann werden mit jedem Wechsel in

$30 \mathrm{Zu}$ diesen beiden Annahmen siehe Marx (1894, 166f und 170). Bortkiewicz (1907a, 21) verwies als erster darauf, daß beide Identitäten nicht gleichzeitig erfüllt sein können; eine allgemeine Darstellung bietet Seton (1956/57). Zur Übersicht über die Diskussion um das Transformationsproblem siehe Heinrich (1988) sowie Howard und King (1992).

31 Wie Heinrich (1991, 47-51) zeigt, stand Ricardo im Unterschied zu Marx gar nicht vor dem Problem, die Arbeitswerte in Produktionspreise zu verwandeln, weil er von vornherein in Kategorien von Produktionspreisen dachte.

32 In seiner frühen Kritik an der Marxschen Preistheorie hat Bortkiewicz (1907a, 16) bereits auf diesen Mangel hingewiesen. 
der Einkommensverteilung, beispielsweise bei einem Anstieg der Profitrate, neue relative Preise entstehen; folglich wird der Wert eines Güterbündels steigen oder fallen, obgleich es seinem Umfang und seiner Zusammensetzung nach gleich bleibt und sich auch die verwendete Produktionstechnik nicht ändert. Solche verteilungsinduzierten Umbewertungen treten sowohl im Modell der interdependenten Produktionsstöme auf, wie es Marx, Walras, Leontief und Sraffa ihren Untersuchungen zugrundelegten, als auch im Modell der linearen Produktionsströme, das von Ricardo, Böhm-Bawerk und Wicksell benutzt wurde.

Wir behandeln im folgenden die Profitrate als jene unabhängige Variable, die herauf- oder herabgesetzt wird; sodann werden die Preise in Lohneinheiten gemessen, so daß gilt: $w=1$. Sowohl aus den Gleichungen [1] und [2] des Marxschen Modells als auch aus den Gleichungen [5] und [6] des Modells der linearen Produktionsströme erhält man für die relativen Preise eine Funktion der Form:

$$
\mathrm{p} / \mathrm{q}=\mathrm{f}\left(\mathrm{r}, \mathrm{x}, \mathrm{y}, \mathrm{x}_{1}, \mathrm{x}_{2}, \mathrm{~L}_{1}, \mathrm{~L}_{2}\right)
$$

In beiden Fällen hängt das Preisverhältnis der beiden Güter Maschine und Tuch nur noch von den bekannten technischen Produktionsbedingungen nämlich von den physischen Einsatzmengen und den Ausbringungsmengen - und der exogenen Profitrate ab. Mit jedem Wechsel in der Profitrate wird sich ein neues Preisverhältnis einstellen; ${ }^{33}$ daher werden die Wertgrößen der Aggregate: Kapital, Einkommen, Profit und Lohnsumme variieren, wann immer die Profitrate steigt oder fällt, obgleich die physischen Mengen in jedem dieser Warenkörbe gleichgeblieben sind. ${ }^{34}$ Sowohl die Marxsche als auch die neoklassische Theorie von Böhm-Bawerk, Jevons und Wicksell führen die gleiche unzulässige Voraussetzung ein, indem sie eine bestimmte Menge der eingesetzten Produktivgüter durch einen Kapitalwert darstellen, von dem angenommen wird, daß seine Größe ausschließlich mit dem Umfang der verwendeten Produktionsmittel variiert. Diese Annahme ist falsch. Wie Sraffas Arbeit gezeigt hat, wird eine gleichbleibende Menge von Produktionsmitteln jedesmal neu bewertet, wenn die Profitrate steigt, so daß der Kapitalwert bei gleichbleibenden physischen Mengen ebenfalls wechseln muß. Ein solcher Kapitalwert, der als Ausdruck einer Menge interpretiert wird, findet als wesentliche Größe Eingang in die weitere Argumentation. So benutzt Marx eine aus der Arbeitswertrechnung erhaltene Verwertungsrate, um die Gleichgewichtspreise zu ermitteln, und die oben angeführten neoklassischen Ökonomen versuchen, mit Hilfe der Grenzpro-

33 Für eine Ökonomie mit beliebig vielen Gütern siehe Pasinetti (1975, 102-104).

34 Wird eines der genannten Aggregate zur Normierung des Preissystems verwendet (so wie oben die Lohnsumme), dann bleibt sein Preisausdruck aus rein definitorischen Gründen konstant, was nicht im Widerspruch zu der eben gemachten Aussage steht. 
duktivität des Kapitals die Profitrate zu determinieren. Dabei changiert die dem Kapital zugedachte Rolle zwischen einer physischen Menge und einer Wertgröße. ${ }^{35}$ In dieser Tradition stehen auch die üblichen Lehrbuchmodelle, in denen ein aggregierter Kapitalwert in eine makroökonomische Produktionsfunktion eingesetzt wird. Die verteilungsinduzierten Umbewertungen der Waren machen solche Versuche aber zunichte.

Dieses Ergebnis ist folgenreich: Der Zusammenhang zwischen einer Änderung der Einkommensverteilung und den Mengeneffekten am Gütermarkt und am Arbeitsmarkt wird zerrissen; solche Mengeneffekte können nicht mehr vorhergesagt werden. Ricardo und Marx, Walras und die Nachfolger, Böhm-Bawerk und Wicksell, Keynes und Sraffa - sie sitzen alle im gleichen Boot. Daß die Beschäftigung zunähme, wenn der Reallohnsatz sinkt, ist sowenig zwingend wie die Behauptung, daß ein geringerer Zinssatz das Aktivitätsniveau der Ökonomie anheben werde, weil der Umfang der Geldvorschüsse wachse. Man kann davon ausgehen, daß beide Aussagen im allgemeinen falsch sind. Ganz gleichgültig auf welchem Beschäftigungsniveau die Ökonomie produziert, eine Verteilungsänderung wird zuerst einmal ein neues System der relativen Preise hervorbringen. Ein Geldvorschuß von 100 DM kann unter Umständen bei einer hohen Zinsrate einen größeren Beschäftigungseffekt haben als ein Vorschuß von $150 \mathrm{DM}$ bei einer niedrigeren Rate, eine gleichbleibende Technik und konstante Skalenerträge vorausgesetzt.

In den jüngeren Theorien - sei es die moderne allgemeine Gleichgewichtstheorie oder die neoricardianische Theorie Sraffas - werden formal konsistente Lösungen für die Einkommensverteilung und die Preisbestimmung gefunden, allerdings hinterlassen sie die offene Frage nach den Faktoren, die das Niveau der Produktion und der Beschäftigung bestimmen. In Sraffas Modell wird vom Aktivitätsniveau der Ökonomie abstrahiert; seiner Theorie der Preisbestimmung genügt ein beliebiges Produktionsniveau. Demgegenüber kann die neoklassische Theorie des allgemeinen Gleichgewichts zwar den Umfang der Produktion und die Höhe der Beschäftigung erklären, aber sie gelangt zu diesem Ergebnis, weil sie schon alles in die Voraussetzungen des Modells hineinlegt: in die Erstausstattung, in die Produktionsverfahren und die Präferenzen. Existieren mehrere Gleichgewichtslösungen, so bleibt auch die Lage des ökonomischen Systems offen. Aus dem Blickwinkel der neoklassischen Ökonomik sind alle wirtschaftlich relevanten Fragen auf die Allokation knapper Ressourcen gerichtet, und die Allokationslösung des Arbeitsmarktes liegt darin, daß der Reallohnsatz einen je gegebenen Bestand an Arbeitskräften in einen freiwillig beschäftigten und einen freiwillig arbeitslosen Teil trennt.

35 In geradezu idealtypischer Reinheit siehe dazu Clark (1899, 121 und 159). 
Betrachtet man den Marxschen Beitrag zur Preis-, Verteilungs- und Kapitaltheorie, so wird deutlich, daß er keineswegs die Sonderstellung einnimmt, welche ihm von seinen Gegnern und Anhängern zugedacht wird. Zum einen haben Marx und eine Reihe von neoklassischen Ökonomen wie Walras, Jevons, Böhm-Bawerk und Wicksell wesentliche Begriffe und ein bestimmtes methodisches Vorgehen von Ricardo übernommen. Zum anderen teilen jene Autoren Probleme der Kapital- und Verteilungstheorie, die ebenfalls auf Ricardos Schrift zurückgehen. Es entbehrt nicht einer gewissen Ironie, wenn Kritiker der Marxschen Werttheorie ihm genau jenen Fehler vorhielten, der ihnen selbst unterlaufen war. Böhm-Bawerk (1884, 39498) beispielsweise hob den Widerspruch zwischen dem System der Arbeitswerte und dem System der Produktionspreise hervor, den Marx durch ein 'dialektisches Gewebe von Ausflüchten' habe zu verdecken gesucht, und er selbst erlitt mit einer Verteilungstheorie Schiffbruch, worin der Profit als Grenzprodukt eines aggregierten Kapitals abgeleitet wurde. Der Trugschluß lag darin, daß jene Kritiker ein Problem der Marxschen Kapitaltheorie, nämlich das Transformationsproblem, als ein spezielles Problem der Arbeitswerttheorie ansahen, welches tatsächlich nur der besondere Fall des Aggregationsproblems ist und ihren eigenen Theorien der Preisbestimmung und der Verteilung sowie den später auf dieser Grundlage entwickelten Theorien der Beschäftigung, die sich auch heute noch vornehmlich in Lehrbüchern finden, den Boden entzieht.

Nach Lage der Dinge ist Marxens Kritik der politischen Ökonomie noch zu schwach. Sraffas Einleitung zu einer Kritik der ökonomischen Theorien hat vernichtend gewirkt, wobei diese Ergebnisse auch von kritischen Vertretern der neoklassischen allgemeinen Gleichgewichtstheorie akzeptiert werden. ${ }^{36}$ Ricardos curious effect, der am Anfang dieser Entwicklung stand, ist zu einem disasterous effect herangewachsen: Der kritische Gehalt der Kapitaltheorie liegt darin, daß mit solchen disaggregierten Modellen simplifizierende makroökonomische Vorstellungen über den Zusammenhang zwischen Verteilung und Beschäftigung vernichtet werden. Oder wie Joan Robinson über das Modell Sraffas sagt: »The function of pure logic is to liberate us from nonsense, not to tell us what we ought to believe« (Robinson $1965,181)$.

36 Siehe dazu Bliss (1975), der im Kapitel 4 (insbesondere S.80-85) auf die Paradoxien der Kapitaltheorie eingeht und im Kapitel 8 die aggregierte Produktionsfunktion einer kritischen Analyse unterzieht; vgl. auch Hahn (1982). 


\section{Literatur}

Bliss, C. J. (1975): Capital theory and the distribution of income, Amsterdam, Oxford

Böhm-Bawerk, Eugen v. (1884): Kapital und Kapitalzins. I. Abteilung: Geschichte und Kritik der Kapitalzinstheorien, Jena 1921

Böhm-Bawerk, Eugen v. (1888): Kapital und Kapitalzins. II. Abteilung: Positive Theorie des Kapitals, Bde.1 und 2, Jena 1921

Bortkiewicz, Ladislaus v. (1907a): Wertrechnung und Preisrechnung im Marxschen System. Zweiter und dritter Aufsatz, in: Archiv für Sozialwissenschaft und Sozialpolitik, Bd.25, S.10-51 und S.445-488

Bortkiewicz, Ladislaus v. (1907b): Zur Berichtigung der grundlegenden theoretischen Konstruktion von Marx im dritten Band des 'Kapital', in: Jahrbücher für Nationalökonomie und Statistik, Bd. 34, S.319-335

Cameron, Burgess (1952): The labour theory of value in Leontief models, in: Economic Journal, vol. 62, pp.191-197

Clark, John Bates (1899): The distribution of wealth, New York 1965

Dmitriev, Vladimir K. (1898): David Ricardos Werttheorie. Versuch einer strengen Analyse, in: Bertram Schefold (Hrsg.): Ökonomische Klassik im Umbruch, Frankfurt 1986

Dobb, Maurice (1973): Wert- und Verteilungstheorien seit Adam Smith, Frankfurt 1977

Eatwell, John (1987): Walras' theory of capital, in: Palgrave Dictionary of Economics, pp.868-872, London 1987

Heinrich, Michael (1988): Was ist die Werttheorie noch Wert? Zur neueren Debatte um das Transformationsproblem und die Marxsche Werttheorie, in: PROKLA 72, S.15-38

Heinrich, Michael (1991): Die Wissenschaft vom Wert. Die Marxsche Kritik der politischen Ökonomie zwischen wissenschaftlicher Revolution und klassischer Tradition, Hamburg

Howard, M.C./ King, J.E. (1989/1992): A history of Marxian Economics, 2 vols., London.

Jevons, William S. (1871): The theory of political economy, London 1931

Keynes, John M. (1933): [Manuskript: The distinction between a co-operative economy and an entrepreneur economy], Collected Writings, vol. 29, pp.76-87, London 1979

Keynes, John M. (1936): Die allgemeine Theorie der Beschäftigung, des Geldes und des Zinses, Berlin 1974

Kurz, Heinz D.; Salvadori, Neri (1995): Theory of production. A long-period analysis, Cambridge

Lange, Oskar (1935): Marxian Economics and modern economic theory, in: Review of Economic Studies, vol.2, S.189-201

Leontief, Wassily W. (1938): The significance of Marxian economics for present-day theory, in: The American Economic Review, vol. 28, 1938, Supplement. Papers and Proceedings of the Fiftieth Annual Meeting of the American Economic Association, pp.1-9

Levine, David (1995): Wealth and freedom. An introduction to political economy, Cambridge

Marshall, Alfred (1890): Principles of Economics, London 1982

Marx, Karl (1863-67): Ökonomische Manuskripte 1863 - 1867. Teil 2. In: MEGA, II. Abt., Bd. 4.2

Marx, Karl (1867): Das Kapital. Bd. 1, Der Produktionsprozeß des Kapitals, MEW 23

Marx, Karl (1885): Das Kapital, Bd. 2, Der Zirkulationsprozeß des Kapitals, MEW 24

Marx, Karl (1894): Das Kapital, Bd. 3, Der Gesamtprozeß der kapitalistischen Produktion, MEW 25

May, Kenneth (1949/50): The structure of classical value theories, in: Review of Economic Studies, vol. 17, S.60-69

Meek, Ronald Lindley (1956): Studies in the labour theory of value, 2.Auflage London 1973

Meek, Ronald Lindley (1977): A plain person's guide to the transformation problem, in: ders.: Smith, Marx, \& after. Ten essays in the development of economic thought, London 1977

Morishima, M. (1973): Marx's economics, Cambridge

Morishima, M. (1974): Marx in the light of modern economic theory, in: Econometrica, vol. 42, S.611-632

Morishima, M. (1977): Walras' economics. A pure theory of capital and money, Cambridge

Morishima, M. (1989): Ricardo's economics: a general equilibrium theory of distribution and growth, Cambridge 
Morishima, M./ Seton, F. (1961): Aggregation on Leontief matrices and the labour theory of value, in: Econometrica, vol. 29, S.203-220

Neuman, John v. (1945/46): A model of general economic equilibrium, in: Review of Economic Studies, vol. 13, pp.1-9

Nurkse, Ragnar (1934/35): The schematic representation of the structure of production, in: Review of Economic Studies, vol. 2, pp.232-44

Pasinetti, Luigi L. (1975): Vorlesungen zur Theorie der Produktion, Marburg 1988

Pasinetti, Luigi L. (1993): Structural economic dynamics. A theory of the economic consequences of human learning, Cambridge

Ricardo, David (1816-18): Letters 1816-1818. The works and correspondence of David Ricardo, vol. VII, Cambridge 1952

Ricardo, David (1817): On the principles of political economy and taxation, in: The works and correspondence of David Ricardo, Vol. I, edited by Piero Sraffa with collaboration of M.H. Dobb, Cambridge 1986

Robinson, Joan (1942): An Essay on Marxian economics, London

Robinson, Joan (1965): A reconsideration of the theory of value, in: dies.: Collected Economic Papers, vol. III, Oxford 1965

Robinson, Joan (1979): The disintegration of economics, in: dies.: Collected Economic Papers, vol. V, Oxford 1979

Samuelson, Paul A. (1957): Wages and interest: a modern dissection of Marxian economic models, in: American Economic Review, vol.47, S.884-912

Samuelson, Paul A. (1959): A modern treatment of the Ricardian economy, in: Quarterly Journal of Economics, vol. 73, pp.1-35 und pp.217-231

Samuelson, Paul A. (1971): Understanding the Marxian notation of exploitation: a summary of the so called transformation problem between Marxian values and competitive prices, in: Journal of Economic Literature, vol. 9, S.399-431

Samuelson, Paul A. (1974): Marx as a mathematical economist, in: Horwich, George; Samuelson, Paul A. (eds); Trade, stability and macroeconomics: Essays in honor of Lloyd A. Metzler, New York, S.269-307

Schumpeter, Joseph A. (1942): Kapitalismus, Sozialismus und Demokratie, Tübingen 1987

Seton, F. (1957): The »transformation problem«, in: Review of Economic Studies, vol.24, S.149160

Smith, Adam (1776): An inquiry into the nature and the causes of the wealth of nations, edited by R.H. Campell, A.S. Skinner and W.B. Todd, 2 Vols., Oxford 1976

Sraffa, Piero (1951): Introduction, in: The works and correspondence of David Ricardo, vol. 1, edited by Piero Sraffa with the collaboration of M.H. Dobb, Cambridge

Sraffa, Piero (1960): Warenproduktion mittels Waren. Einleitung zu einer Kritik der ökonomischen Theorie, Frankfurt 1976

Steedman, Ian (1977): Marx after Sraffa, London 1977

Sweezy, Paul (1942): Theorie der kapitalistischen Entwicklung, Frankfurt 1970

Walras, Léon (1874): Elements of pure economics, Philadelphia 1984

Wicksell, Knut (1901): Vorlesungen über Nationalökonomie auf der Grundlage des Marginalprinzips, Bd.1, dt. Jena 1913, Reprint Aalen 1969 